\title{
Electronic Speed Controller Based Quad Copter Controlled by Hand Gestures
}

\author{
G. Ashwini, J. Aparnapriya
}

\begin{abstract}
To control a Quad copter by means of simply the use of hand gestures.It allows us to recommend a way to perform Human computer Interface.The concept is to extract an antique techniques of controlling a Quad copter using joysticks, computer controlled and so on. with more intuitive approach, that is, through controlling the Quad copter with the assist of hand gestures, for the user to make it greater user friendly,this makes the user not to study different instructions to control a Quad copter, where it can simply work on the hand gestures. Here we present an approach to achieve the illustrated idea by employing transmission of instruction through a Bluetooth module. A simple gyroscope helps inhelps in tracking hand gesture illustration. Command dedication corresponding to shown gestures and acting movement respectively.
\end{abstract}

Key words:Arduino NANO,Electronic Speed Controller (ESC), Gyroscope sensor.

\section{INTRODUCTION}

Topic we are going to deal here is that A quad copter which is flying is controlled by hand gesture .Gesture is a state where it refers to any physical movement of body it may be hand movement or force movement.

In this paper we are controlling the quad copter using hand gesture or movement and a gyroscope sensor is used to recognize this hand movement as a result the quad copter is controlled. [1]. This makes the poilet easy to control the quad copter using hand guestures.

gyroscope sensor ac knowledges a selected hand moment and transmit it to the ground station and in line with the unique hand movement, the Arduino code interprets the vital movement which the quad copter plays similar to such hand gesture[2]. This paper can be improved in many methods:Toachive custom velocity limit, the capacity to reassign gesture'sto distinct drone functions avoiding abstacles and so on.

\section{II.GYROSCOPE SENSOR}

A gyroscope is a sensor used for navigation and size of angular speed in fig(1). Gyroscopes are to be had which can calculate rotational speed in 1, 2, or 3 guidelines. 3-axis gyroscopes are regularly executed with a 3-axis accelerometer to offer a complete 6 diploma-of-freedom movement monitoring tool.

Gyroscopes have advanced from mechanical-inertial spinning gadgets which include rotors, axles, and gimbals to diverse incarnations of virtual and optical devices. Exploits somephysical property is allowing the system to detect it based on rotational speed for some axis.

Revised Manuscript Received on September 14, 2019.

G. Ashwini, AnuragGroup of Institutions, Hyderabad, Telangana, India.(Email: ashwiniece@cvsr.ac.in)

J. Aparnapriya, AnuragGroup of Institutions, Hyderabad, Telangana, India.(Email: aparnaece@cvsr.ac.in)
There are three kinds of gyroscope: Vibrating structure Gyroscope, Rotary (classical) gyroscopes Optical Gyroscopes.

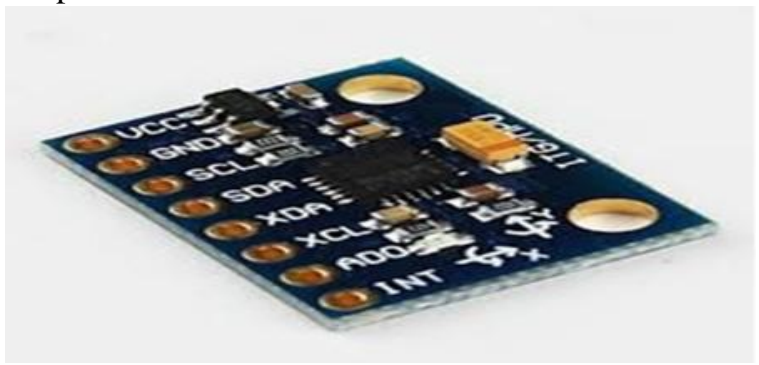

Figure 1: MPU 6050 Gyroscope

Here, we have used MPU 6050 Gyro Accelerometer which falls into the category of classical gyroscopes.

\section{DESIGN APPROACH}

In this papaer, we address the body of the quad copter as Ground station circuitas its circuit consists of the Master module of HC 05 is the Slave module of the Bluetooth and is addressed as the Control circuit for Bluetooth module.

The Ground station circuit consists of

- $\quad$ Arduino NANO

- $\quad$ MPU 6050 Accelerometer-Gyroscope

- $\quad$ Electronic Speed Controller (ESC)

- HC 05 Bluetooth module

- DJI 2212 920KV Brushless Motor

Arduino NANO: The Arduino Nano is a bread board friendly board and also small .It is based on ATmega168 \&ATmega328.It works with a mini-B USB cable as it does not have DC electricity connections external regulated power supply is $5 \mathrm{~V}$.

MPU 6050 Gyro and Accelerometer: The MPU6050 has an embedded three-axis micro electro mechanical system MEMS gyroscope And also accelerometer with three axis MEMS. level converter circuit collectively with the MPU6050 sensor. The functions encompass I2C interface, built in sixteen-bit ADC, digital output temperature sensor and compatibility of $3.3 \mathrm{~V}$-five. $0 \mathrm{~V}$ voltage stage.

Electronic Speed Controller (ESC):An electronic circuit that controls the velocity and also used to exchange the change of electric motor direction [3]. And also a dynamic brake .They are mostly used on radio controlled models ,frequently used in brushless vehicles basically working on three phase powered electricity. 
The electronic speed controller is put into the control channel at receiver channel itself. [4]. HC 05 Bluetooth module: Blutooth is designed generally for serial connection setup , where it is serial port protocol module (SPP).generally Bluetooth is wireless connection setup.To establish a good wireless connection or communication the HC-05 Bluetooth module can be used as both for master and slave configuration[4]. DC Motor is a Brushless DC electric motor called electronically commutated cars (ECMs, EC vehicles) are synchronous motors.

\section{BLOCK DIAGRAM}

The setup in fig(2) consists of Arduino Uno Board, Load Cell(TAL201), Smoke Sensor (MQ2),FireSensor(IR),LoadCell Amplifier(HX711), GPRS module(SIM800), Buzzer, LCD display(16*2), Power Supply Unit. All the components are interfaced to Arduino board which has ATmega328p microcontroller but load cell is interfaced by using a load cell amplifier as the output of load cell is in few millivolts. The inputs from all sensors are taken and given to arduino board where it performs certain operation and result is given through GPRS, buzzer, LCD.The ARDUINO Uno is a microcontroller board based at the ATmega328p. It has 14 digital input/output pins 6 analog inputs, We either need to connect it to a computer using a USB cable or power it with an AC-to-DC adapter.The Arduino circuit acts as an interface among the software program application and the hardware part.

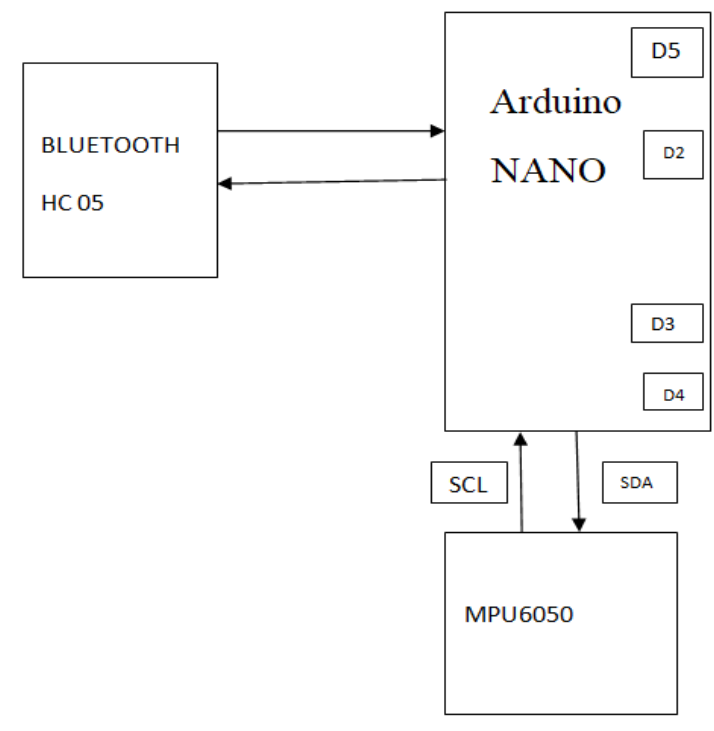

Figure 2: Block Diagram of Hand Gesture Module

\section{RESULTS}

The control circuit of the gyroscopic sensor captures the hand guestures, which sends the information to the ground station in fig(5). The SCL at the ground station translates the gesture after which the talker or publisher approach takes place. The hand guesture is translated to the message on to the quad copter for communication by publisher method to publish.The technique of the quad copter receives the message. the Arduino further translates the relayed message after which instructions of the quad copter to carry out the proper action primarily based at the hand gesture fig(4). The
DJI 2212 920KV Brushless Motor: The 920Kv Brushless

hand gestures relayed are transformed to linear and angular displacements and stored in an array, a displacement is taken and converted in to robot operating system message to be published on the way to result in the desired motion of the quad copter in terms of pitch yaw and roll.

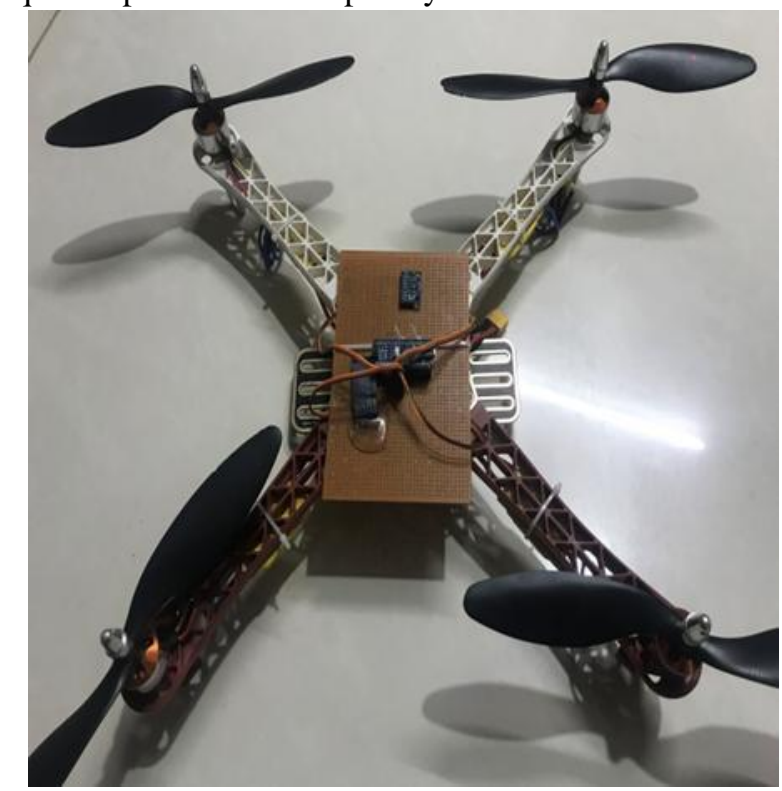

Figure 3: Set up of drone

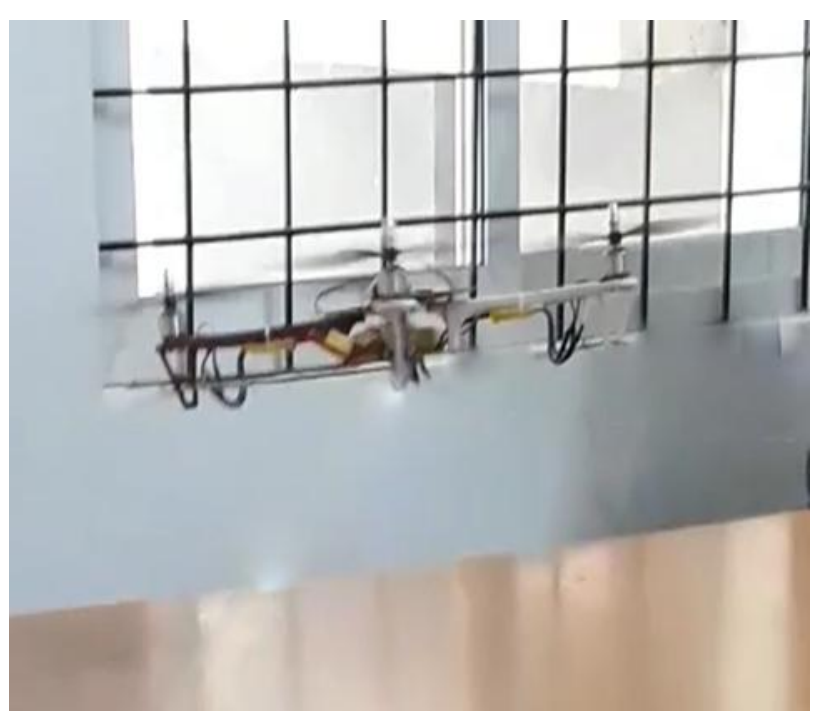

Figure 4: Test result

\section{CONCLUSION}

LEAP movement Controller, capable of move the DRONE via the usage of hand motion. The drone responds to any hand gesture and makes a moment consequently. We have been capable of make the drone flip consistent with sure hand gesture this is over again identified by way of using the 2 stereo cameras and 3 IR LEDs. consequently, it is able to be concluded that with the assist of the leap movement Controller, we're able to use the AR DRONE to carry out numerous responsibilities which includes aerial videos, performing acrobatic responsibilities[5]. The Leap can be taught to apprehend more hand gestures .

Published By: 


\section{FUTURE SCOPE}

The user can limit the maximum speed to a manageable limit that toggle an outdoors mode that off limits the maximum speed or toggles the indoors mode. For easy usage and for the user to have more control. The drone can is capable of detecting certain tags that come to it programmed to follow the user.The drone can follow the user who placed the tags on him/her and gesture .As for pointing at a place for the drone to go there, the direction and the orientation relative to the drone may be calculated alongside statistics from the boundaries avoidance gadget. After that the right commands are despatched to the drone, the drone then flies to the location.

\section{REFERENCES}

1. 1.M Achtelik, T Zhang, K Kuhnlenz, M Buss, "Visual Tracking And Control A Quadcopter Using A Stereo Camera System And Inertial Sensors", Proceeding (ICMA), August 2009.

2. 2. Intelligent Flight Control of an Autonomous Quadrotor University of Ottawa, Canada, pp. 580, January 2010.

3. 3. Lei Zhang, Tianguang Zhang, Haiyan Wu, Alexander Borst, KoljaKuhnlenz, "Visual Flight Control of a Quadrotor using Bio inspired Motion Detector" in Institute of Automatic control Engineering (LSR), Germany:Technische University at Munchen, February 2012.

4. 4. A.A Mian, W Daobo, "Non Linear Flight Control Strategy for an Under Actuated Quadrotor Aerial Robot", Proceeding of the IEEE International Conference on Networking Sensing and control (ICNSC), 2008.

5. 5. Oywind Magnussen, KjellEivind, Skjonhaug, "Modeling Design and Experimental Study for A Quadcopter System Construction", University of Agder Department of Engineering Faculty of Technology and Science, 2011.

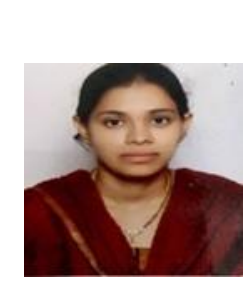

\section{AUTHOR PROFILE}

Ashwinirao.Tuniki received the B.Tech degree in Electronics and Instrumentaion engineering from theJNTUH University, Hyderabad, India, in 2010, and the M.Tech.in Embedded Systems from JNTUH University, Hyderabad, India in 2015.

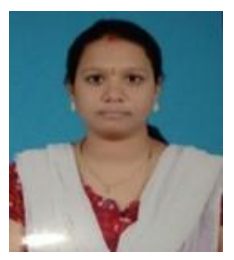

J.Aparnapriya received B.Tech degree in Electronics and communication engineering fom JNTUH university,Hyderabad,India,in 2012 and M.Tech in embedded systems from JNTUH university,Hyderabad, India in 2014. 